\title{
Patients' Beliefs Regarding Dental Health Promotion
}

\author{
Maria Dubielecka, Ewa Rusyan, Aneta Zduniak, \\ Agnieszka Mielczarek \\ Medical University of Warsaw, Warszawa, Poland
}

\author{
Anna Ratajska, Magdalena Lewandowska \\ Kazimierz Wielki University, \\ Bydgoszcz, Poland
}

\begin{abstract}
Objectives: The aim of the present study was to assess patients' perceptions regarding dental caries prophylaxis. Methods: The study sample comprised 170 male and female subjects $(F=96, M=74)$ aged between 18 and 65 years randomly selected from urban (the $\mathrm{U}$ group, $n=110$ ) and rural (the $\mathrm{R}$ group, $n=60$ ) areas. The study examined patients' beliefs regarding follow-up dental visits and professional dental prophylaxis (PDP). Results: The survey revealed that over $60 \%$ of the respondents from both rural and urban areas believed that the dental practitioner should decide whether performing an in-office PDP is necessary. There were significant differences between the rural $(\mathrm{R})$ and urban $(\mathrm{U})$ study samples in how the respondents valued dental check-ups. The subjects from the $\mathrm{R}$ group declared that one dental visit per year should be performed, unlike the $\mathrm{U}$ group subjects who postulated less frequent dental visits. There were no significant differences between the study groups regarding payment decisions for PDP.
\end{abstract}

Keywords: attitude to health, patient acceptance of health care, dental survey

\section{Introduction}

Dental caries remains an on-going health problem. Its management today requires that the patient correctly understand such notions as "monitoring of the carious process" and "non-invasive treatment" (Evans, Pakdaman, Dennison, \& Howe, 2008). It is also necessary that the patient should adhere to principles of health promotion. Oral health behaviours involve twice daily tooth brushing with fluoride toothpaste, flossing, mouth-rinsing and using other dental products. At the same time, it is essential that the patient attend regular check-ups at least once a year so that the provider can tailor treatment plans and ways to prevent health issues in the future to the patient's needs, such as scaling, polishing, and professional application of fluoride preparations. To make dental prophylaxis effective for patients, dental staff should take into consideration patients' beliefs regarding professional dental prophylaxis, and their willingness to co-operate with the dentist. Relevant studies in literature show that the patient's place of residence (a village, city) may have an impact on access to dental health care (Bhagavatula, Xiang, Szabo, Eichmiller, Kuthy, \& Okunsen, 2012).

The study attempted to assess patients' beliefs regarding dental check-ups and professional dental prophylaxes.

Maria Dubielecka, Ph.D., Department of Conservative Dentistry, Medical University of Warsaw.

Ewa Rusyan, Ph.D. med., Department of Conservative Dentistry, Medical University of Warsaw.

Aneta Zduniak, Msc., Department of Conservative Dentistry, Medical University of Warsaw.

Agnieszka Mielczarek, Ph.D., Associate Professor, Department of Conservative Dentistry, Medical University of Warsaw.

Anna Ratajska, Ph.D., Institute of Psychology, Kazimierz Wielki University.

Magdalena Lewandowska, MA., Clinical Communication Centre, Kazimierz Wielki University. 
The following research questions were set up:

(1) Are urban residents more interested in professional caries prevention than residents of rural areas?

(2) Who, in the opinion of the urban and rural respondents, should make the decision about treatment of caries: the dentist or the patient? and

(3) Is there any difference of opinion between urban and rural respondents regarding measurable value of preventive dental prophylaxis.

\section{Material and Method}

The study group comprised 170 male and female subjects $(F=96, M=74)$ aged between 18 and 65 years from urban (the $\mathrm{U}$ group, $n=110$ ) and rural (the $\mathrm{R}$ group, $n=60$ ) areas from the Mazovian province randomly selected in a multilayered draw. The study groups were balanced in relation to gender (test $\chi^{2} p=0.78$ ) and age (test $\chi^{2} p=0.78$ ). The study was based on a questionnaire prepared by the authors and examined patients' beliefs regarding dental caries prevention. The respondents were asked to complete a questionnaire that consisted of 12 questions from three subject areas:

(a) Beliefs regarding the need of dental check-ups and professional dental prophylaxes recommended by the dentist (frequency of dental attendance);

(b) Beliefs regarding measurable value of professional dental prophylaxis; and

(c) Beliefs regarding own ability to plan, implement and follow the dentist's recommendation about ways to prevent health issues.

Prior to completing the questionnaires, the respondents were informed about the aim of the study and also about the nature and significance the professional dental prophylaxis has for overall health. Consent for the study was obtained from the Bioethics Committee of the Medical University of Warsaw.

\section{Statistical Analysis}

The study results were analysed using "Statistica 7.1" by StatSoft. To analyse the results, the Pearson's $\chi^{2}$ test was used, and $p<0.05$ was considered significant.

\section{Results}

As for the questions concerning patients' beliefs regarding the frequency of dental visits that help maintain healthy teeth, the respondents selected one of the three answers:

(1) I believe that if I frequently attend dental check-ups (at least once a year), I will avoid dental problems.

(2) I believe that attending dental check-ups once every two years, or less often, is enough to avoid dental problems.

(3) I don't know how often I should visit a dentist for a check-up to avoid dental problems.

The results are presented in Table 1.

More than half of the respondents residing in rural areas (55\%) were convinced that frequent dental check-ups have a positive effect on maintaining oral health, unlike urban residents (37\%), who failed to see a need for visiting a dentist so often. The analysis of the results revealed statistically significant differences between both groups of respondents.

Further questions in this section concerned patients' beliefs about access to professional dental prophylaxes (PDP) including the financial aspect. The respondents selected one out of the five available 
answers that best reflected their views on the effect and significance of these procedures on oral health.

(4) I believe that undergoing professional dental prophylaxis is the best option only if it is refunded by the National Health Fund (NFZ).

(5) I believe it is proper option to undergo dental prophylaxis one has to pay for.

(6) I believe that one should pay only for dental treatment and I am not going to undergo professional dental prophylaxis that is not free of charge.

(7) I believe that professional dental prophylaxis is ineffective and expensive.

The results are presented in Table 2. The statistical analysis has not revealed significant differences between the study groups with regard to the subjects' views on whether it is right to bear the financial burden of check-ups and professional dental prophylaxes. The highest percentage of the respondents both in the U group (53.64\%) and the R group (38.33\%) accepted the payment for professional dental prophylaxis.

Table 1

Number and Percentage of the Respondents (Urban/Rural) with Different Views Regarding the Frequency of Dental Control Visits to Maintain Oral Health

\begin{tabular}{llllll}
\hline \multirow{2}{*}{ Response category } & \multicolumn{3}{c}{ Urban } & \multicolumn{3}{c}{ Rural } & \\
\cline { 2 - 6 } & $N$ & $\%$ & $N$ & $\%$ & $p=0.026$ \\
\hline Often, at least once a year & 41 & 37.27 & 33 & 55.00 & $p=0.0002^{*}$ \\
Once every two years or less often & 56 & 50.91 & 13 & 21.67 & $p=05$ \\
No opinion & 13 & 11.82 & 14 & 23.33 & $p=0.05$ \\
\hline
\end{tabular}

Table 2

Number and Percentage of the Respondents (Urban/Rural) with Different Views on Undergoing Dental Procedures with Regard to Their Financial Aspect

\begin{tabular}{llrrrc}
\hline \multirow{2}{*}{ Response category } & \multicolumn{3}{c}{ Urban } & \multicolumn{3}{c}{ Rural } \\
\cline { 2 - 5 } & $N$ & $\%$ & \multicolumn{1}{c}{$N$} & $\%$ & \\
\hline Only if free of charge, covered by the NFZ insurance & 18 & 16.33 & 19 & 31.67 & $p=0.14$ \\
Paid care & 59 & 53.64 & 23 & 38.33 & \\
It is more appropriate to invest in therapeutic procedures only & 15 & 13.64 & 9 & 15 & $p=0.76$ \\
Ineffective and expensive & 18 & 16.37 & 9 & 15 & \\
\hline
\end{tabular}

Another group of questions concerned patients' views on whether professional dental prophylaxis is necessary or not.

When asked, "Who should decide about the need for dental check-ups and professional dental prophylaxis - the dentist or the patient?", the majority of the respondents from the U group (68.18\%) and a similar number of the respondents from the R group (61.67\%) found the doctor's decision superior. Dentist as a professional has adequate knowledge of the patient's oral health and is able to set up an individual plan of dental appointments for the patient. More than $31 \%$ of the urban respondents and over $38 \%$ of rural respondents were of a different opinion and believed that the decision lies with the patient. The results are presented in Table 3 .

Statistical analysis revealed no significant differences between the urban and rural respondents (test $\chi^{2} p=$ $0.39)$.

The final group of questions concerned patients' views on being reminded about scheduled appointments. The respondents could choose one out of three opinions regarding appointment reminders sent by the dental practice staff (e-mail, text message, phone call). 
(a) I believe that reminding the patient about the follow-up visit is necessary;

(b) I believe that reminding the patient about the follow-up visit is unnecessary. The patient will keep the scheduled appointment if the visit is important to him/her; and

(c) I believe that reminding the patient about the follow-up visit is unnecessary. Reminders make me feel uncomfortable and forced to attend.

The results are presented in Table 4.

Table 3

Number and Percentage of Respondents (Urban/Rural) Presenting Different Views on Who Should Decide about the Need for Check-ups and Professional Dental Prophylaxis

\begin{tabular}{|c|c|c|c|c|c|}
\hline \multirow{2}{*}{ Response category } & \multicolumn{2}{|c|}{ Urban } & \multicolumn{2}{|c|}{ Rural } & \multirow{2}{*}{$p$} \\
\hline & $N$ & $\%$ & $N$ & $\%$ & \\
\hline Patients make decisions based on their individual needs and time available & 35 & 31.82 & 23 & 38.33 & \multirow{2}{*}{$p=0.39$} \\
\hline Dental professionals make decisions based on patients' dental needs & 75 & 68.18 & 37 & 61.67 & \\
\hline
\end{tabular}

Table 4

Number and Percentage of Respondents (City/Village) Presenting Different Views on the Need to Remind the Patient about the Follow-up Appointment

\begin{tabular}{|c|c|c|c|c|c|}
\hline \multirow{2}{*}{ Response category } & \multicolumn{2}{|c|}{ Urban } & \multicolumn{2}{|c|}{ Rural } & \\
\hline & $N$ & $\%$ & $N$ & $\%$ & \\
\hline Reminding is necessary & 52 & 47.27 & 19 & 31.67 & \\
\hline $\begin{array}{l}\text { Reminding is unnecessary because patients remember about their } \\
\text { appointments, or feel forced }\end{array}$ & 58 & 52.73 & 41 & 68.33 & 0.099 \\
\hline
\end{tabular}

While urban residents declared that reminding patients about their dental appointments is necessary. Rural residents considered patient reminders unnecessary. The analysis revealed no statistically significant differences between the two study groups (test $\chi^{2} p=0.099$ ).

\section{Discussion}

Effective preventive treatment in dentistry, as in any other field of medicine, largely depend on patients' understanding and acceptance of the importance of those interventions (Crocombe, Stewart, Brennan, Slade, \& Spencer, 2012). While treatment plans do not generally raise major objections in patients, plans concerning preventive procedures, such as follow-up visits, changes to dietary habits or oral hygiene are not always accepted by them. Studies conducted in the Netherlands demonstrated that contemporary dental patients are more aware of their rights and may display a more critical attitude towards proposed methods of treatment they want to negotiate. The traditional paternalistic model of the dentist-patient interaction has undergone a transformation. Patients are now far more active players in the treatment process, which may be partly attributed to easier access to current medical knowledge. The more the patient knows about the treatment options, the greater their involvement and willingness to participate in the decision-making process about the choice of particular treatment. Eijkman (Schouten, Hoogstraten, \& Eijkman, 2002) postulates that this observation poses a new challenge for dental practitioners. Doctors have to learn to communicate with their patients more effectively in recommending specific procedures as only half of patients, according to research, comply with doctor's instructions (Eijkman \& Visser, 2001). 
Patient's place of residence (urban/rural) is one of the key factors determining the broadly understood "lifestyle" that impacts patients' general health, including oral health (Brennan \& Spencer, 2005; Freeman, 1999). Since access to dental care varies depending on place of residence, the authors of this study expected to observe significant differences in attitudes towards dental health promoting procedures among residents of these communities. The survey revealed that a significantly lower percentage of urban residents were convinced that dental health could be maintained if check-ups take place at least once a year. Different results were obtained from studies on patients aged 22-44 years in Australia and the United States. The results show a statistically lower interest in professional dental prophylaxis among rural populations against urban residents (Brennan, Spencer, \& Slade, 1996; Skillman, Doesher, Mouradian, \& Brunson, 2010). In the United Kingdom, studies on a representative population sample of patients demonstrated that the majority of the subjects expressed the need for dental check-ups and professional dental prophylaxis (PDP) at 6- and 12-month intervals. Respondents who had the treatments performed less often - every 24 months - expressed their dissatisfaction with a lack of social acceptance resulting from insufficient aesthetics of their teeth (the appearance of teeth, bad breath) (Jone, Macfarlane, Milsom, Ratcliffe, Wyllie, \& Tickle, 2013). In the present studies, rural residents statistically less often decided to pay for the PDP in comparison with urban residents. Similar results were obtained in Swedish studies on 800 subjects from rural and urban communities. The studies revealed that most respondents were not planning to undergo regular dental examinations if these were to be paid for (Ostberg, Ericsson, Wennström, \& Abrahamsson, 2010). Also a survey on a group of 11,000 adults in the UK showed that $26 \%$ of respondents deemed the cost of dental treatment to be crucial in deciding to undergo treatment; $12 \%$ of those surveyed decided to abandon treatment (Hill, Chadwick, Freeman, O'Sullivan, \& Murray, 2013). According to Schouten, Hoogstraten, and Eijkman (2002), weighing treatment costs against the effectiveness of an intervention is a very important factor determining patient's decisions about whether to undergo treatment. Polish studies by Borysewicz-Lewicka and Wochna-Sobańska (2007) indicate that the reason for missing regular dental appointments among $18 \%$ of rural residents and $21 \%$ of people residing in large cities was attributed to financial issues and being unaware of the importance of dental attendance $(16 \%$ and $17 \%$, respectively). In the same studies, rural residents reported that prevention of caries (7\%) and the need for a check-up (27\%) motivated them to see a dentist, while for urban residents the results were $11 \%$ and $34 \%$, respectively (Borysewicz-Lewicka \& Wochna-Sobańska, 2007).

The data on oral health status obtained in the Monitoring of Oral Health of Polish Population show that over $60 \%$ of patients from the Mazovian province paid for their dental services, in comparison with less than $20 \%$ individuals solely dependent on the public health care services (Jodkowska, Wierzbicka, \& Szatko, 2010). The results of the present study concern adult patients' beliefs regarding professional dental prophylaxis and dental check-up. Sources of oral health knowledge include popular science literature, TV and radio broadcasts, friends and family, with the Internet topping the list now. Studies confirm, however, that the information obtained directly from the dental team is deemed the most reliable by the patient (Hugoson, Koch, Göthberg, Helkimo, Lundin, \& Norderyd, 2005). Literature shows that authors of some reports used the Control Preferences Scale, typically used so far for patients seeking general medical help, for dental patients. It is believed that differentiating patients' attitudes as active, cooperative and passive can also be useful in dental management. Research to date shows that patients' passive approach to treatment decision-making, one in which the decision is to be made by the healthcare team, may be due to patients' trust in their doctor or lack of relevant knowledge (Chapple, Shah, Caress, \& Kay, 2003). Results from the present study revealed that Polish 
patients from both rural and urban areas recognized the doctor as the person who decides about implementing professional dental prophylaxis. Similar observations were made by other authors who indicated that some patients, despite medical knowledge and the ability to make autonomous decisions, rest the ultimate decision about the treatment with the doctor (Schouten, Hoogstraten, \& Eijkman, 2003; Woods, 2007).

The analysis of the present research show that the practice of reminding patients about the need for a follow-up dental visit was perceived by $21 \%$ of rural and over $12 \%$ of urban residents as a form of exerting pressure to ensure the appointment takes place. This may be due to communication problems between doctors and patients, patients' mistrust, or unclear and too professional language used by a dentist. The key to success in dental caries prevention is to comply with dental health advice that is defined and modified depending on the clinical condition of a patient (Curtis, Warren, Pollicino, Evans, Schwarz, \& Sbaraini, 2011). Therefore, it is crucial to understand the mechanisms underlying patients' decisions regarding preventing oral health problems, with a particular focus on caries prevention, which still remains a problem in Polish population.

Research in this area concerns mainly patients' decision-making regarding a specific dental condition (Kiesler \& Auerbach, 2006). Authors admit that in cases involving pain or immediate medical intervention, patients' expectations of doctors may differ. Likewise, the communication process may take a different course compared to a situation in which the treatment has been planned (Schouten et al., 2003). There is not enough research to evaluate patients' attitudes to decision-making about professional dental prophylaxis.

\section{Conclusion}

The patient's sense of responsibility for the treatment outcome is of a particular value in the course of a therapeutic process. The closer the patient cooperates with the doctor, the better the treatment outcome, and the easier it is for the patient to accept potential difficulties. Mutual trust is required to build good rapport between the patient and the doctor. Besides, the patient must understand the objectives of the preventive and therapeutic dental interventions. The way patients respond to dentists' recommendations regarding follow-up visits, hygiene and dietary habits may differ. The present research shows that the awareness of rural and urban respondents regarding in-office treatments aiming to prevent dental problems appears to be insufficient. Therefore it is all the more important to emphasize the need for a good doctor-patient communication especially in the case of undertaking preventive dental procedures phased over time. Identifying patients' beliefs regarding preventive dental prophylaxis cannot be overestimated so that their significance and real health benefit are understood. Being knowledgeable about communication issues enables dentist to be more effective in persuading their patients to make informed decisions.

\section{References}

Bhagavatula, P., Xiang, Q., Szabo, A., Eichmiller, F., Kuthy, R., \& Okunsen, Ch. (2012). Rural-urban differences in dental service use among children enrolled in a private dental insurance plan in Wisconsin: Analysis of administrative data. $B M C$ Oral Health, 12, 58-65.

Borysewicz-Lewicka, M., \& Wochna-Sobańska, M. (2007). Dental health-promoting behavior of "A month of totally healthy smile" program. Czas. Stomatol. LX, 6, 377-383.

Brennan, D. S., \& Spencer, A. J. (2005). The role of dentist, practice and patient factors in the provision of dental service. Community Dent Oral Epidemiol., 33, 181-195.

Brennan, D. S., Spencer, A. J., \& Slade, G. D. (1996). Provision of public dental service in urban, rural and remote locations. Community Dent Health, 13(3), 157-162. 
Chapple, H., Shah, S., Caress, A. L., \& Kay, E. L. (2003). Exploring dental patients preferred role in treatment decision-making-A novel approach. British Dental Journal, 194, 321-327.

Crocombe, L., Stewart, J., Brennan, D., Slade, G., \& Spencer, A. (2012). Is poor access to dental care why people outside capital cities have poor oral health? Aust. Dent J., 57, 477-485.

Curtis, B., Warren, E., Pollicino, C., Evans, R. W., Schwarz, E., \& Sbaraini, A. (2011). The Monitor Practice Program: Is non-invasive management of dental caries in private practice cost-effective? Australian Dent J., 56, 48-55.

Eijkman, M. A. J., \& Visser, A. Ph. (2001). Dental health education: From education to informed decision making. PEC, 42, 101-104.

Evans, R. W., Pakdaman, A., Dennison, P. J., Howe, E. L. C. (2008). The Caries Management System: An evidence-based preventive strategy for dental practitioners: Application for adults. Australian Dent J., 53, 83-92.

Freeman, R. (1999). A psychodynamic understanding of the dentist-patient relationship. Br. Dent J., 186(10), 503-506.

Hill, K., Chadwick, B., Freeman, R., \& O’Sullivan, M. J. (2013). Adult Dental Health Survey (2009): Relationships between dental attendance patterns, oral health behavior and the current barriers to dental care. BDJ, 214(1), 25-32.

Hugoson, A., Koch, G., Gőthberg, C., Helkimo, A. N., Lundin, S. A., \& Norderyd, O. (2005). Oral health of individuals aged 3-80 years in Jönköping, Sweden during 30 years (1973-2003). Review of findings on dental habits and knowledge of oral health. Swed. Dent J., 29(4), 125-138.

Jodkowska, E., Wierzbicka, M., \& Szatko, F. (2010). Results of nationwide epidemiological surveys of oral health and treatment needs in children ages 6 and 12 and adults ages 35-44 in Poland (ISBN 978-83-7637-188-7).

Jones, C., Macfarlane, T., Milsom, K., Ratcliffe, P., Wyllie, A., \& Tickle, M. (2013). Patient perceptions regarding benefits of single visit scale and polish: a randomized controlled trial. BMC Oral Health, 13, 50.

Kiesler, D. J., \& Auerbach, S. M. (2006). Optimal matches of patient preferences for information, decision-making and interpersonal behavior: Evidence, models and interventions. PEC, 61, 319-341.

Ostberg, A. L., Ericsson, J. S., Wennström, J. L., \& Abrahamsson, K. H. (2010). Socio-economic and lifestyle in relation to priority of dental care in a Swedish adolescent population. Swed. Dent J., 34(2), 87-94.

Schouten, B. C., Hoogstraten, J., \& Eijkman, M. A. J. (2002). Dutch dental patients on informed consent: Knowledge, attitudes, self-efficacy and behavior. PEC, 46, 47-54.

Schouten, B. C., Hoogstraten, J., \& Eijkman, M. A. J. (2003). Patient participation during dental consultations: The influence of patients' characteristics and dentists' behavior. Community Dent. Oral Epidemiol, 31, 368-377.

Skillman, S. M., Doesher, M. P., Mouradian, W. E., \& Brunson, D. K. (2010). The challenge to delivering oral health services in rural America. J. Public Health Dent, 70 Suppll, 49-57.

Woods, C. D. (2007). A difficult patient: A psychodynamic perspective. CDA J., 35(3), 186-191. 